\title{
Microsatellite instability is linked to loss of hMLH1 expression in advanced gastric cancers: lack of a relationship with the histological type and phenotype
}

\author{
Tsutomu Mizoshita ${ }^{1}$, Tetsuya Tsukamoto ${ }^{1}$, Xueyuan CaO $^{1}$, Takafumi Otsuka ${ }^{1}$, Seiji Ito $^{2}$, Emiko Takahashi $^{3}$, \\ Shigeo Nakamura ${ }^{3}$, Tsuneya Nakamura ${ }^{4}$, Yoshitaka Yamamura ${ }^{2}$, and Masae Tatematsu ${ }^{1}$ \\ ${ }^{1}$ Division of Oncological Pathology, Aichi Cancer Center Research Institute, 1-1 Kanokoden, Chikusa-ku, Nagoya 464-8681, Japan \\ ${ }^{2}$ Department of Gastroenterological Surgery, Aichi Cancer Center Hospital, Nagoya, Japan \\ ${ }^{3}$ Department of Pathology and Clinical Laboratories, Aichi Cancer Center Hospital, Nagoya, Japan \\ ${ }^{4}$ Department of Gastroenterology, Aichi Cancer Center Hospital, Nagoya, Japan
}

\begin{abstract}
Background. It has been suggested that the prevalence of microsatellite instability (MSI) is high in intramucosal differentiated gastric cancers with gastric foveolar phenotypic expression, and that these tumors are prone to lose their glandular structures and progress to undifferentiated-type lesions. To test this hypothesis, we examined the relationships among human MutL homologue 1 (hMLH1) expression (which is linked to MSI), the phenotype, and the histological type in patients with advanced and intramucosal gastric cancer.

Methods. We analyzed hMLH1 expression by immunohistochemistry in 70 advanced and 30 intramucosal gastric cancers with histological evaluation and assessment of the phenotype, and Cdx 2 expression determined by immunohistochemistry. The MSI status was also examined in 20 cases.

Results. Thirteen (18.6\%) advanced and 5 (16.7\%) intramucosal gastric cancers were judged to be hMLH1negative. In the advanced cases, no association was observed between the histological type and the phenotype and loss of hMLH1. In the intramucosal cases, MUC5AC expression was observed in all 5 hMLH1-negative differentiated-type cancers. However, no hMLH1-negative lesions were detected in the intramucosal undifferentiated cancers $(0 / 14 ; P<0.05$ vs differentiated types). In the advanced cases, MSI-positivity $(\mathrm{MSI}+)$ and loss of hMLH1 expression did correlate $(P<$ 0.0001), while no association was observed between MSI +, histological type, and phenotype.

Conclusion. Our data support the hypothesis that, phenotypically, some MSI-positive differentiated gastric cancers of gastric foveolar phenotypic expression may easily change, from gastric to intestinal phenotypic expression, also changing, histologically, from differentiated to undifferentiated type with progression.
\end{abstract}

Key words Microsatellite instability · hMLH1 · Phenotype Intramucosal gastric cancers · Advanced gastric cancers

Offprint requests to: T. Tsukamoto

Received: November 18, 2004 / Accepted: March 23, 2005

\section{Introduction}

Microsatellite instability (MSI) is characterized by length mutations in tandem oligonucleotide repeats. This type of mutation occurs in a large subset of human tumors [1], and is believed to be caused by altered DNA mismatch repair (MMR) [2]. With regard to MMR genes, it is known that hypermethylation of the hMLH1 MMR gene promoter diminishes the expression of this enzyme in human tumors [3]. Such hypermethylation of hMLH1 is strongly associated with MSI in gastric cancers [4], and loss of hMLH1 expression is, in fact, extremely frequent in MSI-positive gastric cancers [5].

Several reports have indicated that it is possible to analyze the phenotypic expression of each gastric cancer cell using gastric and intestinal epithelial cell markers, independent of the histological classification [6-15]. Endoh et al. [16,17] demonstrated a high prevalence of MSI in differentiated-type gastric tumors showing distinctive features of gastric foveolar epithelium, which are prone to loss of their glandular structure and progression to undifferentiated-type tumors [18], whereas, Shibata et al. [19] found no relation between MSI and the phenotype in early differentiated gastric carcinomas. However, to our knowledge, there have been no studies analyzing the links between MSI and the stages and histological types of gastric cancer, in terms of gastric and intestinal phenotypic expression.

In this study, we therefore analyzed hMLH1 expression by immunohistochemistry, in 70 advanced and 30 intramucosal gastric cancers. Histological evaluation was done by hematoxylin and eosin (H\&E) staining, and assessment of the phenotype was carried out by immunohistochemistry. In addition, the MSI status was examined in 20 cases to allow comparisons of the phenotypic expression along with cancer progression. 


\section{Patients, materials, and methods}

\section{Samples and tissue collection}

We examined 70 primary advanced and 30 intramucosal gastric cancers surgically resected at Aichi Cancer Center Hospital between 1994 and 2000 [12,13]. Of the 70 patients with advanced gastric cancers, 41 were men and 29 were women; they ranged in age from 32 to 84 years (mean, $61.8 \pm 10.5$ years). The lesions comprised 32 differentiated and 38 undifferentiated-type cancers, the former found in patients ranging in age from 52 to 84 years (mean, $62.7 \pm 10.1$ years) and the latter in individuals aged from 32 to 83 years (mean, $61.1 \pm 10.9$ years). Of the 30 patients with intramucosal gastric cancers, 20 were men and 10 were women; they ranged in age from 23 to 74 years (mean, $59.5 \pm 11.2$ years). The lesions comprised 16 differentiated and 14 undifferentiated-type cancers, the former found in patients ranging in age from 44 to 74 years (mean, $62.8 \pm 8.1$ years) and the latter in individuals aged from 23 to 72 years (mean, $55.9 \pm 13.2$ years). Histological classification was made according to the Japanese classification of gastric carcinomas [20]. In the patients with advanced disease, the cancers had invaded the subserosa (ss) or the serosa and the peritoneal cavity (se), including the adjacent organs (si). Informed consent for our study was obtained from all participating patients before surgery.

Within 30-40 min after removal of the stomach, the carcinoma tissues and the adjacent non-neoplastic mucosa were carefully sampled, cut into 5-mm squares on ice, and frozen at $-80^{\circ} \mathrm{C}$ for later DNA extraction. Portions of the sampled tissues were fixed in $10 \%$ buffered formalin and then processed to paraffin sections for the confirmation of carcinoma and adjacent non-neoplastic mucosa in hematoxylin-eosin stained sections.

\section{Immunohistochemistry}

Immunohistochemical staining of the paraffin sections was carried out with monoclonal antibodies against the following antigens: hMLH1 (G168-15; 1:200, PharMingen, San Diego, CA, USA); Cdx2 (CDX2-88; 1:50, BioGenex, San Ramon, CA, USA); MUC5AC (CLH2; 1:500, Novocastra Laboratories, Newcastle upon Tyne, UK); MUC6 (CLH5; 1:500, Novocastra Laboratories); MUC2 (Ccp58; 1 :500, Novocastra Laboratories); and villin (12; 1:20000, Transduction Laboratories, Lexington, KY, USA). With regard to gastric phenotypic markers, we used normal gastric mucosa and normal ileum as positive and negative controls, and used these in reverse for the intestinal phenotype. The precise procedures for immunohistochemical techniques were as previously described [8,11-13,21,22]. Briefly, 4- $\mu$ m-thick consecutive sections were deparaf- finized and hydrated through a graded series of alcohols. After the inhibition of endogenous peroxidase activity by immersion in $3 \% \mathrm{H}_{2} \mathrm{O}_{2} /$ methanol solution, antigen retrieval was conducted, for the detection of binding of the above-mentioned antibodies, with $10 \mathrm{mM}$ citrate buffer ( $\mathrm{pH}$ 6.0) in a microwave oven for $10 \mathrm{~min}$ at $98^{\circ} \mathrm{C}$. Sections were incubated with primary antibodies, thoroughly washed in phosphate-buffered saline (PBS), then incubated with biotinylated secondary antibody, followed by treatment with avidin-biotinylated horseradish peroxidase complex (Vectastain Elite ABC kit; Vector Laboratories, Burlingame, CA, USA). Finally, immune complexes were visualized by incubation with $0.01 \% \mathrm{H}_{2} \mathrm{O}_{2}$ and $0.05 \%$ 3,3'-diaminobenzidine tetrachloride (DAB). Nuclear counterstaining was accomplished with Mayer's hematoxylin.

Two independent pathologists (T.M. and T.T.) judged the histology and immunohistochemical staining for the phenotypic markers, Cdx2 and hMLH1. The results of staining for each antibody were evaluated in terms of the percentage of positively stained cancer cells, with $10 \%$ and above considered positive, as previously described [11-13,22,23].

\section{Classification of cancers}

MUC5AC and MUC6 are markers of the gastricepithelial cell phenotype, whereas MUC2 and villin are typical of the intestinal-epithelial cell phenotype $[8,11-$ $13,21]$. Gastric cancers in which more than $10 \%$ of the section area consisted of either gastric or intestinal epithelial cell phenotype were classified as gastric ( $\mathrm{G}$ type) or intestinal (I type) phenotype cancers, respectively. Those which showed both gastric and intestinal phenotypes were classified as gastric and intestinal mixed phenotype (GI type) cancers, while those showing neither gastric nor intestinal phenotype expression were grouped as null type ( $\mathrm{N}$ type) [11-13].

\section{Microsatellite analysis}

We screened for MSI using the primer set BAT-26, which amplifies 26 adenine-mononucleotide repeats in the fifth intron of the $h M S H 2$ gene. It is more than 99.5\% sensitive for detecting MSI-positive (MSI +) tumors, including gastric cancers [16,17,23-27]. Genomic DNAs from frozen tissues were isolated with a DNeasy Tissue Kit (Qiagen, Hilden, Germany). Primers specific for each locus were used to amplify the DNA pairs prepared from tumors and adjacent non-neoplastic mucosa by polymerase chain reaction (PCR). PCR was performed with Titanium Taq PCR kits (Clontech Laboratories, Palo Alto, CA, USA) for 1 cycle of $95^{\circ} \mathrm{C}$ for $2 \mathrm{~min}$, followed by 30 cycles consisting of a denaturation step at $94^{\circ} \mathrm{C}$ for $45 \mathrm{~s}$, an annealing step at $57^{\circ} \mathrm{C}$ 
for $45 \mathrm{~s}$, and an elongation step at $72^{\circ} \mathrm{C}$ for $60 \mathrm{~s}$, in the presence of $0.2 \mathrm{mCi}$ of $\left[{ }^{32} \mathrm{P}\right]$-dCTP, according to the manufacturer's instructions. A final elongation at $72^{\circ} \mathrm{C}$ for $7 \mathrm{~min}$ followed. PCR products were electrophoresed in a denaturing $6 \%$ polyacrylamide gel, containing $8 \mathrm{M}$ urea, at $90 \mathrm{~W}$ for $1.5 \mathrm{~h}[22,23,28,29]$. The gel was then dried on filter paper and subjected to imaging [30]. Bands of abnormal molecular weights of tumor DNA, not observed in background mucosa, were designated as MSI + [16,26].

\section{Statistical analysis}

The data were analyzed by Fischer's exact test or the $\chi^{2}$ test for differences between groups. Survival curves after surgery were drawn using the Kaplan-Meier method. Statistical comparison of survival was performed using the log-rank test. $P$ values of less than 0.05 were considered statistically significant.

\section{Results}

\section{Classification of gastric cancers histologically and phenotypically}

The 70 advanced gastric cancers were classified histologically as 32 differentiated and 38 undifferentiated lesions. The former were clearly subclassified as $8 \mathrm{G}$, $7 \mathrm{GI}, 10 \mathrm{I}$, and $7 \mathrm{~N}$ types phenotypically, using the gastric and intestinal epithelial phenotypic markers (Table 1). Similarly, the 38 undifferentiated cancers could be clearly classified as $8 \mathrm{G}, 11 \mathrm{GI}, 8 \mathrm{I}$, and $11 \mathrm{~N}$ types, on the basis of their phenotype (Table 1 ).

In the 30 intramucosal cases, the cancers were classified histologically as 16 differentiated and 14 undifferentiated lesions. The former were clearly subclassified phenotypically as 2 G, 8 GI, $5 \mathrm{I}$, and $1 \mathrm{~N}$ types (Table 2). Similarly, the 14 undifferentiated cancers were clearly classified as $8 \mathrm{G}, 3 \mathrm{GI}, 1 \mathrm{I}$, and $2 \mathrm{~N}$ types on the basis of their phenotypic expression (Table 2).

\section{The expression of hMLH1 in advanced gastric cancers}

Fifty-seven (81.4\%) and 13 (18.6\%) advanced gastric cancers were judged to be hMLH1-positive and hMLH1-negative, respectively (Table 1). Histologically, the hMLH1-negative lesions were divided into 9 differentiated and 4 undifferentiated types, with no statistically significant link to differentiation, although a trend was suggested $(P=0.072)$. Phenotypically, the hMLH1negative cases were classified as $4 \mathrm{G}, 1 \mathrm{GI}, 5 \mathrm{I}$, and $3 \mathrm{~N}$ types (Figs. 1 and 2), again with no significant association with differentiation $(P=0.32)$.

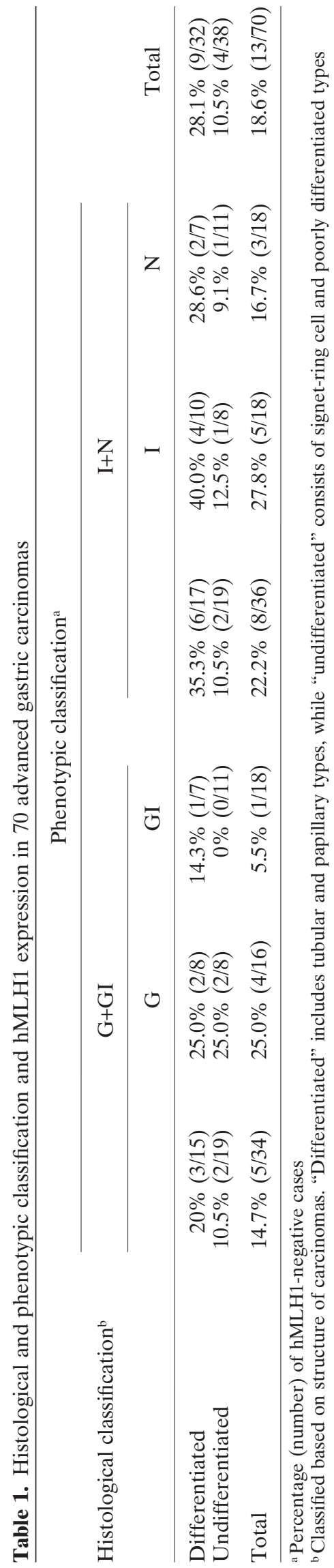






The expression of hMLH1 in intramucosal gastric cancers

Twenty-five (83.3\%) and $5(16.7 \%)$ intramucosal gastric cancers were judged to be hMLH1-positive and hMLH1-negative, respectively (Table 2). Histologically, all 5 hMLH1-negative lesions were differentiated type, with none of the hMLH1-negative lesions being undifferentiated $(* * * P<0.05$ differentiated vs undifferentiated type; Table 2). In the differentiated cases, the expression of MUC5AC, a gastric foveolar phenotypic marker, was judged to be positive in all 5 hMLH1negative lesions, and positivity for MUC5AC tended to be higher in the G+GI phenotypes than in the $\mathrm{I}+\mathrm{N}$ counterpart, although the difference did not reach statistical significance ( $* * P=0.09$; Table 2$)$. In other words in the G+GI phenotypes, there were significantly more hMLH1-negative cases of the differentiated type than of the undifferentiated type $(* P<0.05$; Table 2$)$.

\section{MSI status in advanced gastric cancers}

To cast light on the mismatch repair system, BAT-26, a microsatellite indicator of mononucleotide repeats, was used as a marker for MSI + (Fig. 3). We analyzed the MSI status in 5 hMLH1-negative and 15 hMLH1positive cases. Data on MSI status, hMLH1 expression, and Cdx2 expression are shown in Table 3. All 5 MSI + cases demonstrated loss of hMLH1 expression $(P<$ $0.0001)$. No association was observed between MSI and the histological type or the phenotype. The expression of MUC5AC as a gastric foveolar phenotypic marker was judged to be positive in cases 1 and 4 . Less than $10 \%$ of the section area exhibited MUC5AC in cases 2, 3 , and 5. Cdx2 nuclear staining was not associated with the MSI status.

Postoperative survival analysis of patients with gastric cancer with reference to hMLH1 expression and MSI status

On Kaplan-Meier analysis, the patients with advanced gastric cancers who were hMLH1-negative had a better outcome than those in hMLH1-positive group, but the difference was not significant $(P=0.14$; Fig. 4$)$. The MSI status was not linked with patient survival (data not shown).

\section{Discussion}

Our present data demonstrate that a strong association was observed between MSI + and loss of hMLH1 in advanced gastric cancers, as previously described [4,5], but there was no link between MSI, histological type, 

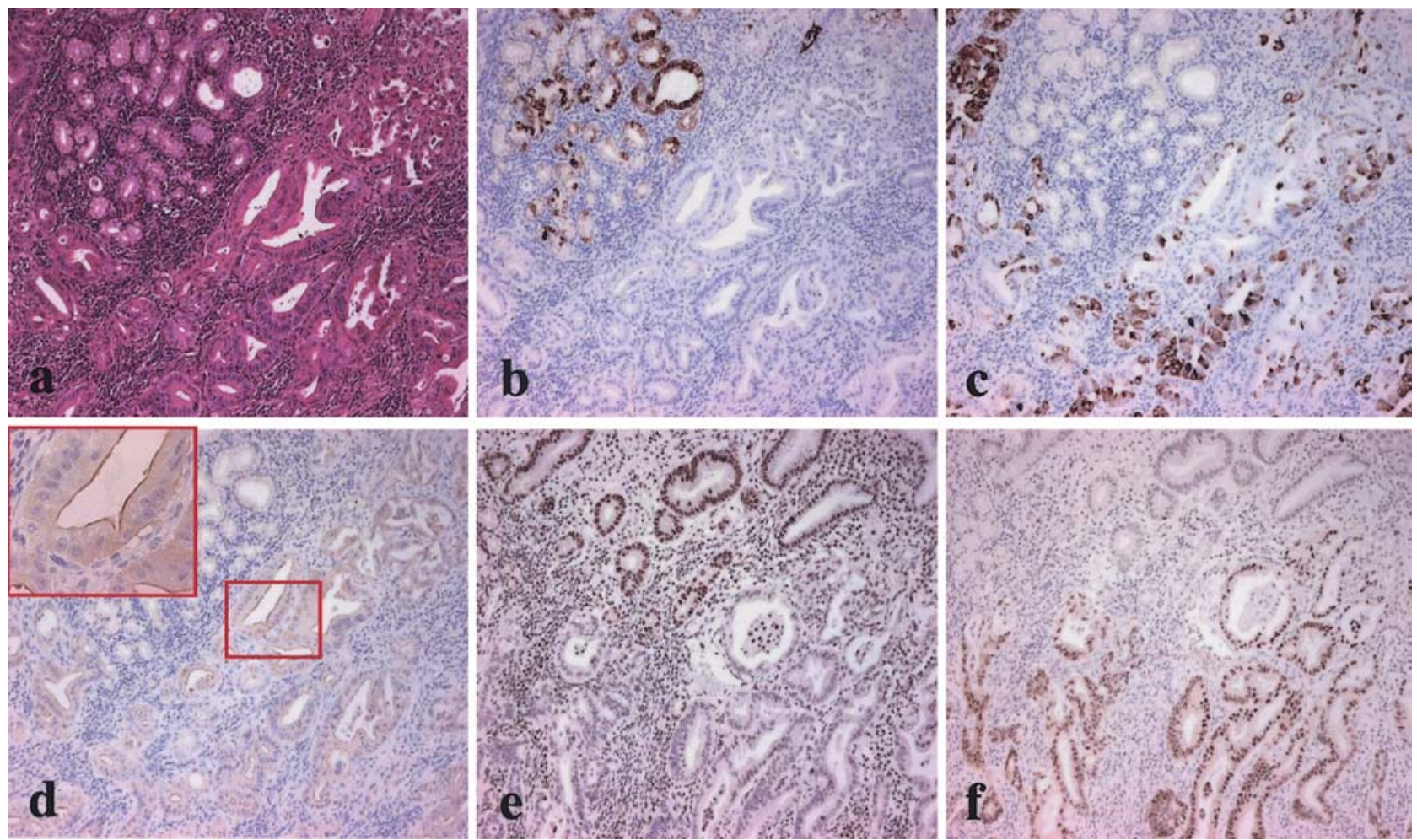

Fig. 1a-f. Moderately differentiated adenocarcinoma of intestinal phenotypic expression (case 3). a H\&E staining. b MUC5AC is present in the cytoplasm of normal gastric mucosa, but not in cancer cells. c MUC2 is positive in the cytoplasm of cancer cells, but not in normal gastric mucosa. d Villin is positive on the luminal surface of cancer cells, but not in normal gastric mucosa. Inset, higher magnification of the cancer cells. e Note, human MutL homologue 1 (hMLH1) nuclear staining is observed in the normal gastric mucosa, but not in carcinoma cells. $\mathbf{f} \mathrm{Cdx} 2$ nuclear staining is apparent in the cancer cells, but not in normal gastric mucosa. (a-f Original $\times 100$; Inset, magnification of red square is $\times 560$ )

Table 3. MSI status, hMLH1 expression, and Cdx2 expression in gastric cancers from 20 patients

\begin{tabular}{|c|c|c|c|c|c|c|c|c|}
\hline Case number & Age (years) & Sex & Depth $^{\mathrm{a}}$ & Histological type & Phenotype & $\mathrm{Cdx} 2$ & hMLH1 & MSI \\
\hline Case 1 & 52 & M & ss & Differentiated & G & - & - & + \\
\hline Case 2 & 52 & M & ss & Differentiated & I & + & - & + \\
\hline Case 3 & 52 & $\mathrm{~F}$ & ss & Differentiated & I & + & - & + \\
\hline Case 4 & 59 & $\mathrm{~F}$ & se & Undifferentiated & $\mathrm{G}$ & - & - & + \\
\hline Case 5 & 72 & M & ss & Undifferentiated & $\mathrm{N}$ & - & - & + \\
\hline Case 6 & 74 & M & se & Undifferentiated & $\mathrm{G}$ & - & + & - \\
\hline Case 7 & 64 & $\mathrm{~F}$ & se & Differentiated & $\mathrm{G}$ & - & + & - \\
\hline Case 8 & 55 & M & ss & Differentiated & GI & + & + & - \\
\hline Case 9 & 65 & $\mathrm{~F}$ & ss & Differentiated & I & + & + & - \\
\hline Case 10 & 66 & M & ss & Differentiated & I & + & + & - \\
\hline Case 11 & 53 & $\mathrm{~F}$ & ss & Differentiated & I & + & + & - \\
\hline Case 12 & 54 & $\mathrm{~F}$ & SS & Undifferentiated & G & - & + & - \\
\hline Case 13 & 67 & M & se & Undifferentiated & $\mathrm{G}$ & - & + & - \\
\hline Case 14 & 67 & M & si & Undifferentiated & $\mathrm{G}$ & - & + & - \\
\hline Case 15 & 70 & $\mathrm{~F}$ & ss & Undifferentiated & GI & + & + & - \\
\hline Case 16 & 43 & $\mathrm{~F}$ & si & Undifferentiated & GI & + & + & - \\
\hline Case 17 & 56 & $\mathrm{~F}$ & se & Undifferentiated & GI & + & + & - \\
\hline Case 18 & 53 & M & si & Undifferentiated & I & + & + & - \\
\hline Case 19 & 67 & M & ss & Undifferentiated & $\mathrm{N}$ & - & + & - \\
\hline Case 20 & 59 & M & si & Undifferentiated & $\mathrm{N}$ & - & + & - \\
\hline
\end{tabular}

G, gastric phenotype; GI, gastric and intestinal phenotype; I, intestinal phenotype; N, null phenotype; MSI, microsatellite instability a The cancers had invaded the subserosa (ss) or the serosa and the peritoneal cavity (se), including the adjacent organs (si) 

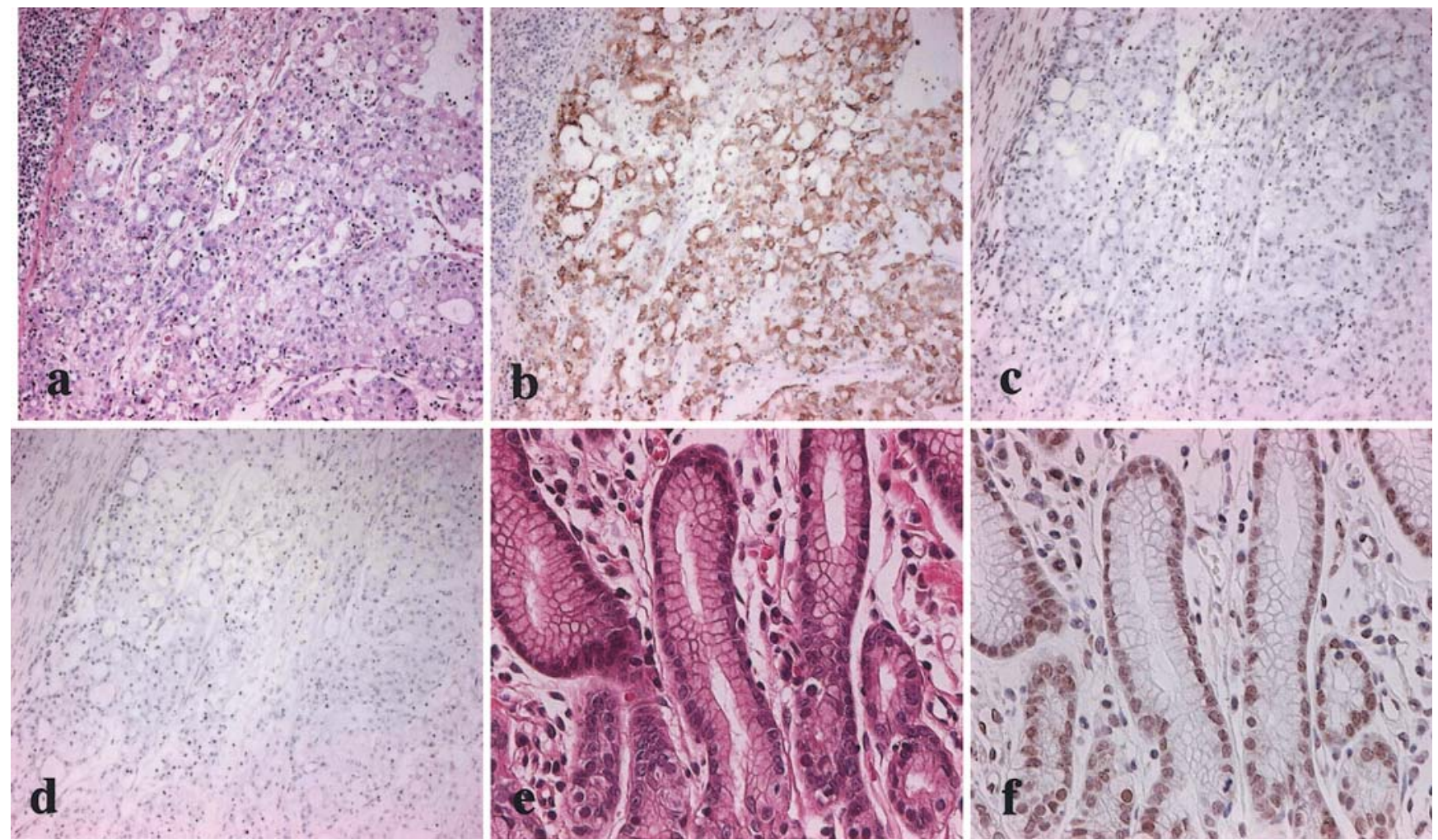

Fig. 2a-f. Poorly differentiated adenocarcinoma featuring gastric foveolar phenotypic expression (case 4). a H\&E staining of tumor areas. b MUC5AC is present in the cytoplasm of cancer cells. c No hMLH1 nuclear staining is observed in cancer cells. d No Cdx2 nuclear staining is apparent in the cancer cells. e H\&E staining of surrounding normal gastric mucosa. f hMLH1 nuclear staining is observed in the normal gastric mucosa. $\mathbf{a}, \mathbf{b}, \mathbf{c}$, and $\mathbf{d}, \times 100$; $\mathbf{e}$ and $\mathbf{f} \times 320$

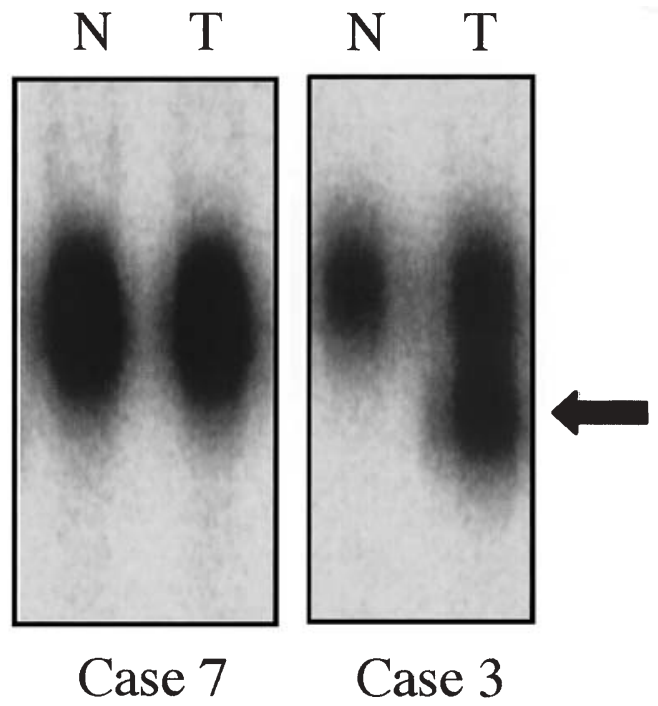

Fig. 3. Microsatellite instability (MSI) analysis, case 3, mobility shift indicating MSI is indicated by the arrow: case 7 shows no mobility shift. $N$, normal DNA; $T$, tumor DNA

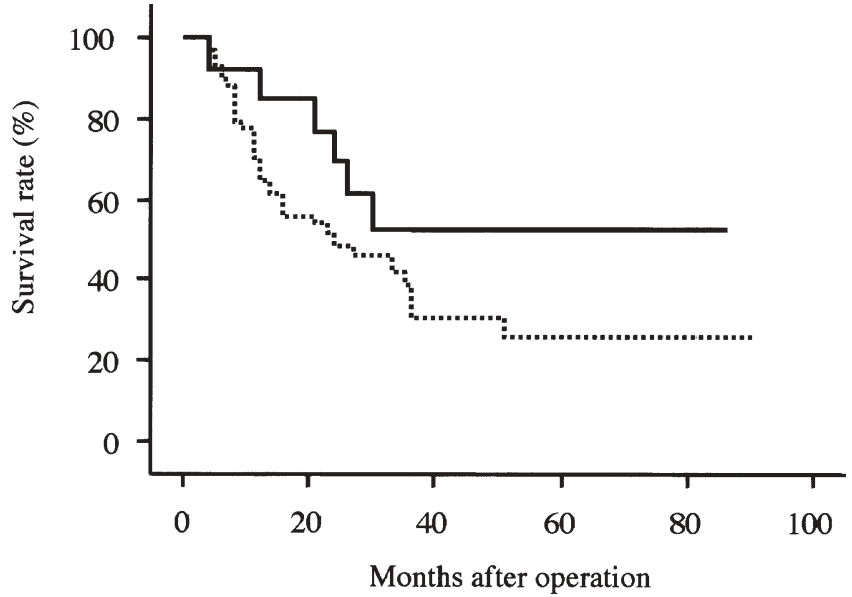

Fig. 4. Postoperative survival curves of patients with advanced gastric cancer with reference to hMLH1 expression. The hMLH1-negative patients (continuous line; $n=13$ ) had a better outcome than the hMLH1-positive group (dotted line; $n$ $=57)$, but the difference was not significant $(P=0.14)$ 
and phenotype in the advanced gastric cancers. Shibata et al. [19], similarly, found no relationship between MSI and phenotype in differentiated gastric carcinomas, although Endoh et al. $[16,17]$ reported an association with gastric foveolar phenotypic expression. This different result may be due to differences between the studies in the stages of gastric cancers examined. We separately evaluated intramucosal cancers and advanced cases invading beyond the subserosa in depth, while most of the cases analyzed by Endoh et al. [16,17] were intramucosal lesions. In fact, in their study, of 17 early differentiated gastric cancers of the gastric foveolar type, $12(70.6 \%)$ were judged to be MSI + , while in 6 advanced differentiated gastric cancers of the gastric foveolar type, only 1 case $(16.7 \%)$ was judged to be MSI +, suggesting the lack of a relationship between MSI + and phenotype in advanced cases [17]. Regarding the I type, they found that, in 11 differentiated gastric cancers, only 1 case with submucosal invasion $(9.1 \%)$ was MSI + [17]. Our data showed that, in the differentiated advanced gastric cancers, 2 (25\%) and 1 (14.3\%) of the $8 \mathrm{G}$ and $7 \mathrm{GI}$ types, respectively, were judged to be hMLH1-negative, while in 10 differentiated advanced gastric cancers of I type, 4 cases $(40 \%)$ were hMLH1-negative (Tables 1 and 3). In addition, of our $2 \mathrm{G}$ and $8 \mathrm{GI}$ intramucosal differentiated-type gastric carcinomas, $2(100 \%) \mathrm{G}$ and $3(37.5 \%)$ GI cases had loss of hMLH1. All 5 hMLH1-negative cases had MUC5AC expression, suggesting an association between gastric foveolar phenotypic expression and MSI. No hMLH1-negative lesions were observed in the intramucosal differentiated gastric cancers of I type. It is well-known that human gastric cancers at an early stage, independent of the histological type, mainly consist of gastric-phenotype malignant cells, while their advanced counterparts tend to have more intestinal-phenotype malignant cells, suggesting a phenotypic shift from gastric to intestinal phenotypic expression during the course of tumor progression $[6,8,10,14,31,32]$. Thus, our data support the hypothesis that some MSI-positive differentiated gastric cancer cells featuring gastric foveolar phenotypic expression may easily change, phenotypically from gastric to intestinal phenotypic expression with progression. However, further studies of a larger number of intramucosal and submucosal/advanced differentiated gastric cancer cases may be needed to clarify any association between the phenotype and MSI/loss of hMLH1.

Our data also demonstrated no relationship between MSI + and histological type in the advanced gastric cancers, while a significant association was observed between differentiated type and loss of hMLH1 in the intramucosal gastric cancers. We need to consider changes in the features of gastric cancer cells with pro- gression, from the viewpoint of the histological type. Regarding the histological type, Endoh et al. [18] have presented evidence that certain differentiated-type gastric carcinomas possess the features of gastric foveolar epithelium, and these are prone to progress to undifferentiated-type carcinomas through the loss of Ecadherin function. Kushima and Hattori [9] have also shown a close relationship between differentiated-type carcinomas with a predominant gastric foveolar epithelial phenotype and progression to undifferentiated type. Of 38 advanced undifferentiated gastric cancers in our study, 4 were judged to be hMLH1-negative (Table 1). Of 5 MSI + cases, 2 were undifferentiated type (Table 3). However, in 14 intramucosal undifferentiated gastric cancers, no hMLH1-negative lesions were observed. We therefore consider the possibility that some MSI-positive differentiated gastric cancers of gastric foveolar phenotypic expression may change from differentiated to undifferentiated type histologically with progression.

Hinoi et al. [33] have reported that loss of Cdx2 expression and MSI are prominent features of large-cell minimally differentiated carcinomas of the colon. However, we could find no relationship between the MSI status and $\mathrm{Cdx} 2$ expression in advanced gastric cancers. Similarly, no association was observed between the expression of hMLH1 and Cdx2. Also, we and others have noted a strong association between $\mathrm{Cdx} 2$ nuclear staining and intestinal phenotypic expression, independent of the histological type, in gastric cancers [11-13,34]. Thus, we consider that altered DNA mismatch repair may have no influence on the intestinal phenotype, including $\mathrm{Cdx} 2$ expression, in gastric cancers.

Our data have shown that, in patients with advanced gastric cancer the hMLH1-negative patients had a better outcome than the hMLH1-positive group, but the difference was not significant $(P=0.14$; Fig. 4). Gastric cancers of microsatellite mutator phenotype had a good prognosis, shown by multivariate analysis [35]. A univariate analysis showed that gastric cancers of microsatellite mutator phenotype showing altered loci had a better prognosis than those showing two or fewer altered loci [36]. Thus, altered DNA mismatch repair may be linked with the prognosis of gastric cancers.

Intestinal metaplasia (IM) is widely thought to be a precancerous lesion for differentiated-type gastric cancers. However, previous studies of the phenotypic expression of individual intestinal metaplastic or stomach cancer cells have pointed to several contradictions to the prevailing paradigm $[6-10,13,37,38]$. With regard to MSI analysis, Tamura et al. [39] have shown that the majority of differentiated adenocarcinomas of the stomach may develop through a de-novo pathway, from the viewpoint of microsatellite alterations. Endoh et al. [16] 
also made it clear that the genetic backgrounds of differentiated-type tumors were quite different among different cellular phenotypes. Frequent MSI and infrequent $p 53$ mutation are observed in tumors of gastric foveolar phenotypic expression, and conversely, infrequent MSI and considerable $p 53$ mutations are detected in tumors with a distinct intestinal cellular phenotype [16]. MSI occurs not only in gastric IM of patients with gastric carcinoma, but also in IM of cancer-free individuals [28]. However, Jin et al. [26] have reported that instability of BAT-26, a sensitive marker for the highfrequency MSI phenotype, was not found in intestinal metaplastic mucosa adjacent to any of a series of gastric carcinomas. Thus, further studies are clearly warranted to clarify whether IM is, indeed, a precancerous lesion.

In conclusion, our data support the hypothesis that, phenotypically, some MSI-positive differentiated gastric cancers of gastric foveolar phenotypic expression may, easily change from gastric to intestinal phenotypic expression, also changing, histologically from differentiated to undifferentiated type with progression.

Acknowledgments The authors thank Dr. Malcolm A. Moore for revision of the scientific English language and Ms. Hisayo Ban for expert technical assistance. This study was supported in part by a Grant-in-Aid for the Third-Term Comprehensive 10-year Strategy for Cancer Control, a Grant-in-Aid for Cancer Research from the Ministry of Health, Labour and Welfare, Japan, and a Grant-in-Aid from the Ministry of Education, Culture, Sports, Science and Technology of Japan.

\section{References}

1. Han HJ, Yanagisawa A, Kato Y, Park JG, Nakamura Y. Genetic instability in pancreatic cancer and poorly differentiated type of gastric cancer. Cancer Res 1993;53:5087-9.

2. Thibodeau SN, French AJ, Roche PC, Cunningham JM, Tester DJ, Lindor NM, et al. Altered expression of hMSH2 and hMLH1 in tumors with microsatellite instability and genetic alterations in mismatch repair genes. Cancer Res 1996;56:4836-40.

3. Kane MF, Loda M, Gaida GM, Lipman J, Mishra R, Goldman H, et al. Methylation of the hMLH1 promoter correlates with lack of expression of hMLH1 in sporadic colon tumors and mismatch repair-defective human tumor cell lines. Cancer Res 1997;57:80811.

4. Fleisher AS, Esteller M, Wang S, Tamura G, Suzuki H, Yin J, et al. Hypermethylation of the hMLH1 gene promoter in human gastric cancers with microsatellite instability. Cancer Res 1999; 59:1090-5.

5. Leung SY, Yuen ST, Chung LP, Chu KM, Chan AS, Ho JC. hMLH1 promoter methylation and lack of hMLH1 expression in sporadic gastric carcinomas with high-frequency microsatellite instability. Cancer Res 1999;59:159-64.

6. Tatematsu M, Furihata C, Katsuyama T, Miki K, Honda H, Konishi Y, et al. Gastric and intestinal phenotypic expressions of human signet ring cell carcinomas revealed by their biochemistry, mucin histochemistry, and ultrastructure. Cancer Res 1986; 46:4866-72.
7. Tatematsu M, Ichinose M, Miki K, Hasegawa R, Kato T, Ito N. Gastric and intestinal phenotypic expression of human stomach cancers as revealed by pepsinogen immunohistochemistry and mucin histochemistry. Acta Pathol Jpn 1990;40:494-504.

8. Tatematsu M, Tsukamoto T, Inada K. Stem cells and gastric cancer. Role of gastric and intestinal mixed intestinal metaplasia. Cancer Sci 2003;94:135-41.

9. Kushima R, Hattori T. Histogenesis and characteristics of gastrictype adenocarcinomas in the stomach. J Cancer Res Clin Oncol 1993;120:103-11.

10. Egashira Y, Shimoda T, Ikegami M. Mucin histochemical analysis of minute gastric differentiated adenocarcinoma. Pathol Int 1999;49:55-61.

11. Mizoshita T, Tsukamoto T, Nakanishi H, Inada K, Ogasawara N, Joh T, et al. Expression of Cdx2 and the phenotype of advanced gastric cancers: relationship with prognosis. J Cancer Res Clin Oncol 2003;129:727-34.

12. Mizoshita T, Inada K, Tsukamoto T, Nozaki K, Joh T, Itoh M, et al. Expression of the intestine-specific transcription factors, Cdx1 and $\mathrm{Cdx} 2$, correlates shift to an intestinal phenotype in gastric cancer cells. J Cancer Res Clin Oncol 2004;130:29-36.

13. Mizoshita T, Tsukamoto T, Inada K, Ogasawara N, Hirata A, Kato S, et al. Immunohistochemically detectable Cdx2 is present in intestinal phenotypic elements in early gastric cancers of both differentiated and undifferentiated types, with no correlation to non-neoplastic surrounding mucosa. Pathol Int 2004;54:392400.

14. Yoshikawa A, Inada K, Yamachika T, Shimizu N, Kaminishi M, Tatematsu M. Phenotypic shift in human differentiated gastric cancers from gastric to intestinal epithelial cell type during disease progression, Gastric Cancer 1998;1:134-41.

15. Tsukamoto T, Mizoshita T, Mihara M, Tanaka H, Takenaka Y, Yamamura Y, et al. Sox2 expression in human stomach adenocarcinomas with gastric and gastric-and-intestinal-mixed phenotypes. Histopathology 2005;46:649-58.

16. Endoh Y, Sakata K, Tamura G, Ohmura K, Ajioka Y, Watanabe $\mathrm{H}$, et al. Cellular phenotypes of differentiated-type adenocarcinomas and precancerous lesions of the stomach are dependent on the genetic pathways. J Pathol 2000;191:257-63.

17. Endoh Y, Tamura G, Ajioka Y, Watanabe H, Motoyama T. Frequent hypermethylation of the hMLH1 gene promoter in differentiated-type tumors of the stomach with the gastric foveolar phenotype. Am J Pathol 2000;157:717-22.

18. Endoh Y, Tamura G, Watanabe H, Ajioka Y, Motoyama T. The common 18-base pair deletion at codons 418-423 of the E-cadherin gene in differentiated-type adenocarcinomas and intramucosal precancerous lesions of the stomach with the features of gastric foveolar epithelium. J Pathol 1999;189:201-6.

19. Shibata N, Watari J, Fujiya M, Tanno S, Saitoh Y, Kohgo Y. Cell kinetics and genetic instabilities in differentiated type early gastric cancers with different mucin phenotype. Hum Pathol 2003; 34:32-40.

20. Japanese Gastric Cancer Association. Japanese classification of gastric carcinoma, 2nd English Edition. Gastric Cancer 1998;1:1024.

21. Tsukamoto $\mathrm{T}$, Inada $\mathrm{K}$, Tanaka $\mathrm{H}$, Mizoshita $\mathrm{T}$, Mihara $\mathrm{M}$, Ushijima $\mathrm{T}$, et al. Down regulation of a gastric transcription factor, Sox2, and ectopic expression of intestinal homeobox genes, $\mathrm{Cdx} 1$ and $\mathrm{Cdx} 2$ : inverse correlation during progression from gastric/intestinal-mixed to complete intestinal metaplasia. J Cancer Res Clin Oncol 2004;130:135-45.

22. Wu CW, Chen GD, Jiang KC, Li AF, Chi CW, Lo SS, et al. A genome-wide study of microsatellite instability in advanced gastric carcinoma. Cancer 2001;92:92-101.

23. Kim HS, Lee BL, Woo DK, Bae SI, Kim WH. Assessment of markers for the identification of microsatellite instability phenotype in gastric neoplasms. Cancer Lett 2001;164:61-8.

24. Myeroff LL, Parsons R, Kim SJ, Hedrick L, Cho KR, Orth K, et al. A transforming growth factor beta receptor type II gene muta- 
tion common in colon and gastric but rare in endometrial cancers with microsatellite instability. Cancer Res 1995;55:5545-7.

25. Zhou XP, Hoang JM, Li YJ, Seruca R, Carneiro F, SobrinhoSimoes M, et al. Determination of the replication error phenotype in human tumors without the requirement for matching normal DNA by analysis of mononucleotide repeat microsatellites. Genes Chromosomes Cancer 1998;21:101-7.

26. Jin Z, Tamura G, Satoh M, Meguro T, Miura T, Hayashi M, et al. Absence of BAT-26 instability in gastric intestinal metaplasia. Pathol Int 2001;51:473-5.

27. Takahashi H, Endo T, Yamashita K, Arimura Y, Yamamoto H, Sasaki S, et al. Mucin phenotype and microsatellite instability in early multiple gastric cancers. Int J Cancer 2002;100:419-24.

28. Leung WK, Kim JJ, Kim JG, Graham DY, Sepulveda AR. Microsatellite instability in gastric intestinal metaplasia in patients with and without gastric cancer. Am J Pathol 2000;156:53743.

29. Bae SI, Park JG, Kim YI, Kim WH. Genetic alterations in gastric cancer cell lines and their original tissues. Int J Cancer 2000; 87:512-6.

30. Shirai N, Tsukamoto T, Yamamoto M, Iidaka T, Sakai H, Yanai $\mathrm{T}$, et al. Elevated susceptibility of the p53 knockout mouse esophagus to methyl- $N$-amylnitrosamine carcinogenesis. Carcinogenesis 2002;23:1541-7.

31. Yamachika T, Inada K, Fujimitsu Y, Nakamura S, Yamamura Y, Kitou $\mathrm{T}$, et al. Intestinalization of gastric signet ring cell carcinomas with progression. Virchows Arch 1997;431:103-10.

32. Bamba M, Sugihara H, Kushima R, Okada K, Tsukashita S, Horinouchi M, et al. Time-dependent expression of intestinal phenotype in signet ring cell carcinomas of the human stomach. Virchows Arch 2001;438:49-56.

33. Hinoi T, Tani M, Lucas PC, Caca K, Dunn RL, Macri E, et al. Loss of CDX2 expression and microsatellite instability are prominent features of large cell minimally differentiated carcinomas of the colon. Am J Pathol 2001;159:2239-48.

34. Almeida R, Silva E, Santos-Silva F, Silberg DG, Wang J, De Bolos $\mathrm{C}$, et al. Expression of intestine-specific transcription factors, CDX1 and CDX2, in intestinal metaplasia and gastric carcinomas. J Pathol 2003;199:36-40.

35. Yamamoto H, Perez-Piteira J, Yoshida T, Terada M, Itoh F, Imai $\mathrm{K}$, et al. Gastric cancers of the microsatellite mutator phenotype display characteristic genetic and clinical features. Gastroenterology 1999;116:1348-57.

36. dos Santos NR, Seruca R, Constancia M, Seixas M, SobrinhoSimoes M. Microsatellite instability at multiple loci in gastric carcinoma: clinicopathologic implications and prognosis. Gastroenterology 1996;110:38-44.

37. Kawachi H, Takizawa T, Eishi Y, Shimizu S, Kumagai J, Funata $\mathrm{N}$, et al. Absence of either gastric or intestinal phenotype in microscopic differentiated gastric carcinomas. J Pathol 2003; 199:436-46.

38. Hattori T. Development of adenocarcinomas in the stomach. Cancer 1986;57:1528-34.

39. Tamura G, Sakata K, Maesawa C, Suzuki Y, Terashima M, Satoh $\mathrm{K}$, et al. Microsatellite alterations in adenoma and differentiated adenocarcinoma of the stomach. Cancer Res 1995;55:1933-6. 